\title{
Implementation of an Artificial Neural Network on the Test Barcelona Workstation As a Predictive Model for the Classification of Normal, Mild Cognitive Impairment and Alzheimer's Disease Subjects Using the Neuronorma Battery
}

\author{
Neus Rivera, ${ }^{1,2}$, Margarita Cabrera-Bean³, Gonzalo Sánchez-Benavides ${ }^{2,4}$, \\ Carles Gallego-González ${ }^{5}$, Jose A. Lupiáñez-Pretel ${ }^{5}$, and Jordi Peña-Casanova ${ }^{1,2}$ \\ ${ }^{1}$ Universitat Autònoma de Barcelona (SPAIN, Barcelona) \\ ${ }^{2}$ Mar Institute of Medical Research Foundation (FIMIM) (SPAIN, Barcelona) \\ 3 Universitat Politècnica de Catalunya (UPC) (SPAIN, Barcelona) \\ 4BarcelonaBeta Brain Research Center, Fundació Pasqual Maragall (SPAIN, Barcelona) \\ ${ }^{5}$ Infoal-ITF (SPAIN)
}

Corresponding Author:

Neus Rivera

Nieves.Rivera@uab.cat

Received: 25 July 2018

Accepted: 9 August 2018

Published: 1 November 2018

Publishing services provided by Knowledge

(c) Neus Rivera et al. This article is distributed under the terms of the Creative Commons

Attribution License, which permits unrestricted use and redistribution provided that the original author and source are credited.

Selection and Peer-review under the responsibility of the Fifth International Luria Memorial Congress Conference Committee.

\section{Abstract}

Objective: To develop and implement an online Artificial Neural Network (ANN) that provides the probability of a subject having mild cognitive impairment $(\mathrm{MCl})$ or Alzheimer's disease (AD).

Method: Different ANNs were trained using a sample of 350 controls (CONT), $75 \mathrm{MCl}$ and 93 AD subjects. The ANN structure chosen was the following: (1) an input layer of 33 cognitive variables from the Neuronorma battery plus two sociodemographic variables, age and education. This layer was reduced to a 15 features input vector using Multiple Discriminant Analysis method, (2) one hidden layer with 8 neurons, and (3) three output neurons corresponding to the 3 expected cognitive states. This ANN was defined in a previous study [28]. The ANN was implemented on the web site www.test-barcelona.com (Test Barcelona Workstation) [9].

Results: When comparing CONT, MCI and AD participants, the best ANN correctly classifies up to $94,87 \%$ of the study participants.

Conclusions: The online implemented ANN, delivers the probabilities (\%) of belonging to the CONT, MCl and AD groups of a subject assessed using the 35 characteristics (variables) of the Neuronorma profile. This tool is a good complement for the interpretation of cognitive profiles. This technology improves clinical decision making.

Keywords: Artificial Neural Network, Probability, Alzheimer disease, Test Barcelona Worksation.

\section{G OPEN ACCESS}




\section{Introduction}

Mild cognitive impairment is the diagnostic term given to subjects who are thought to have a cognitive impairment greater than expected for age and education without an obvious etiology, but sufficiently severe to warrant a diagnosis of dementia [12, 24, 31]. Having $\mathrm{MCl}$ increases the risk of developing a dementia like $A D$.

Neuropsychological assessment is the first key investigation for the diagnosis of $A D$. It is used to document the pattern and degree of deficits in the various domains of cognitive functions such as memory, language, attention, orientation, and executive functions. A comprehensive neuropsychological examination can provide a pattern of impaired/preserved functions that is helpful to early detection of dementia. The selected tests should be adapted and standardized to the studied population in order to properly estimate cognitive and functional performance [23].

To facilitate the diagnostic process and to avoid misdiagnosis, artificial intelligence methods can be used. These adaptive learning algorithms can handle diverse types of medical data and integrate them into categorized outputs [1]. Data from several studies have pointed out the existence of a strong correlation between AD neuropathology and cognitive state. However, because of their highly complex and nonlinear relationship, it has been difficult to develop a predictive model for individual patient classification through traditional statistical approaches. When exposed to complex data sets, ANNs can recognize patterns, learn the relationship of different variables, and address classification tasks [4]. The adaptive systems provide superior accuracy when compared to models of traditional linear statistics [7].

In 2001, Peña-Casanova et al. developed the Neuronorma battery [16]. The objective was to collect normative and psychometric information on a sample of people aged over 49 years, based on 33 neuropsychological tests covering attention, language, visuo-perceptual abilities, constructional tasks, memory, and executive functions. Effects of age, education, and sex were determined. In 2016, Rivera et al. implemented an ANN to provide the probability of a subject having $\mathrm{MCl}$ or $\mathrm{AD}$ using as a feature input vector the Neuronorma battery. As a background, in 2011, Quintana et al. implemented an ANN to classify the same subjects of this study, evaluated with the Test Barcelona neuropsychological battery [25].

\section{Methodology}




\subsection{Subjects}

The study was carried out on 518 participants: 350 CONT, $75 \mathrm{MCl}$, and 93 AD. Participants ranged in age from 50 to 90 years old with years of education from 3 to 18 . The mean ages (standard deviation) of the three groups were 64.96 (9.24), 72.69 (6.64) and 74.42 (7.50) years, respectively. The mean education (standard deviation) of the three groups were 10.59 (5.45), 8.12 (4.86) and 7.62 (4.81) years, respectively. Subjects were recruited and assessed as part of the Neuronorma project [16].

\subsection{Variables}

The following test were used to determine the cognitive state of participants (see in Peña-Casanova, et al, 2009a-f): Verbal span (Digit Span forward and backward) Spanish version [15]; Visuospatial Span (Corsi's Test) from the WAIS-R-NI [10]; Trail Making Test [14, 26]; Symbol Digit Modalities Test [29]; Boston Naming Test [11]; Token Test [6]; Selected test of the Visual Object and Space Perception Battery [30]; Judgment of Line Orientation [2]; Rey-Osterrieth Complex Figure [13, 27]; Free and Cued Selective Reminding Test [3]; Verbal fluency [15]; Stroop Color-Word Interference Test [8]; Tower of London Drexel University version [5]. A total of 33 cognitive variables were used. These variables plus age and education were used as the input dataset to train ANNs.

\subsection{Artificial neural network}

An ANN consists of a system composed by one input layer, one output layer, and one or more hidden layers. In this study ANNs with only one hidden layer were executed. Each layer is composed by nodes, all of them connected through different weights. During a learning or training period the weights are fitted or adjusted, processing each input vector and comparing the output with a corresponding target. In this work the input vector was formed by the neurological test indicators (Neuronorma profile) and the target was the corresponding subject class: CONT, MCI or AD. The experiments carried out were based on feedforward neural networks operating in a supervised learning mode, i.e. a known diagnostic outcome is available during the training period. Once the neural network has been trained it can be used to diagnose new subjects, not included in the initial database formed by the 518 participants 


\subsection{Procedure}

The initial database was divided in three groups or datasets: $70 \%$ to train, $15 \%$ to validate and $15 \%$ to test. In the training phase the network compares the real output with the expected diagnosis (target) and uses the difference to adapt the weights set of the ANN. The training cycle is iteratively repeated until the validation set finds the minimum mean square error (MSE: quadratic difference between the output and the target or expected diagnosis). The optimization of the weights of the network is determined by the minimization of this MSE. So, the validation dataset provides an unbiased evaluation of the ANN that has been fitted on the training dataset.

The optimal neural network architecture was defined using two parameters: the number of hidden neurons, and the dimension of the input vector, also known as hyperparameters of the system. Many experiments were performed with the initial database. To reduce the features of the input vector two methods were proved: Principal Component Analysis (PCA: the objective was to transform the vector into a new dimensionally reduced vector, to emphasize variation and bring out the information existing in the data), and Multiple Discriminant Analysis (MDA: the objective was to project the vector to maximize the differences between classes [samples well differentiated]). Six preprocessed databases were obtained: (1) RS-WR: raw input vector without reduction; (2) RS-PCA: raw input vector with PCA reduction; (3) RS-MDA: raw input vector with MDA reduction; (4) SS-WR: input vector adjusted by age and education without reduction; (5) SS-PCA: input vector adjusted by age and education with PCA reduction; and (6) SS-MDA: input vector adjusted by age and education with MDA reduction.

With these preprocessed databases, different ANNs were trained and validated whose only difference was the number of hidden neurons, and furthermore, all the results were also compared with another classifier, the Linear Discriminant. The test dataset was used to provide the final unbiased evaluation of the best ANN obtained with each preprocessed database.

\section{Results}

The classification error of the neutral database (test dataset) using the two methods to reduce the characteristics of the input vector, PCA and MDA, and without any reduction is shown in Table 1; both by the LD classifier and by the ANN. 
The best trained ANN was composed by 15 input nodes (characteristics were reduced from 35 to 15 by MDA method) and 8 hidden neurons. This ANN was implemented on the Test Barcelona Workstation. Figure 1 shows the Neuronorma profile of a subject with its associated probability of belonging to one of the three cognitive states: Normal Aging (CONT), $\mathrm{MCl}$ and Dementia (AD).

TABLE 1: Classification error of the 3 classes (CONT, MCl and AD) using the 6 preprocessed input databases.

\begin{tabular}{l|c|c|c|c|c|c|}
\multicolumn{2}{c}{ Lineal Discriminant Analysis } & \multicolumn{4}{c|}{ Artificial Neural Network } \\
Database & $\begin{array}{c}\text { Characteristic } \\
\text { reduction }\end{array}$ & $\begin{array}{c}\text { Classification } \\
\text { error }\end{array}$ & Database & $\begin{array}{c}\text { Characteristic } \\
\text { reduction }\end{array}$ & $\begin{array}{c}\text { Hidden } \\
\text { neurons }\end{array}$ & $\begin{array}{c}\text { Classification } \\
\text { error }\end{array}$ \\
\hline SS & PCA & $11.54 \%$ & RS & MDA & 8 & $5.13 \%$ \\
\hline SS & WR & $12.82 \%$ & RS & WR & 6 & $6.41 \%$ \\
\hline SS & MDA & $12.82 \%$ & RS & PCA & 5 & $6.41 \%$ \\
\hline RS & MDA & $14.10 \%$ & SS & PCA & 14 & $12.82 \%$ \\
\hline RS & WR & $15.38 \%$ & SS & WR & 35 & $15.38 \%$ \\
\hline RS & PCA & $16.67 \%$ & SS & MDA & 34 & $17.95 \%$ \\
\hline
\end{tabular}

RS = Raw Score; SS = Scaled Score; PCA = Principal Component Analysis; MDA = Multiple Discriminant Analysis; WR = Without Reduction.

\subsection{Limitations and future studies}

Currently, the ANN implemented on the Test Barcelona Workstation is only useful for Alzheimer's disease suspected cases. Our group has initiated similar studies in patients with multiple sclerosis, vascular dementia and Parkinson's disease.

\section{Conclusions}

This study evaluated the capacity of an online ANN to predict the cognitive state of a subject assessed by the Neuronorma battery. The study shows that the ANN approach is better than linear predictive models. The online ANN is composed of 15 input nodes and 8 hidden nodes. The input vector, compounded of the values of Neuronorma test plus age and education, is previously reduced by MDA method. The classification accuracy achieved is nearly $95 \%$. In conclusion, the results of this study confirm that the online ANN on the Test Barcelona Workstation is an excellent tool to asses clinical decisions. 
(A)

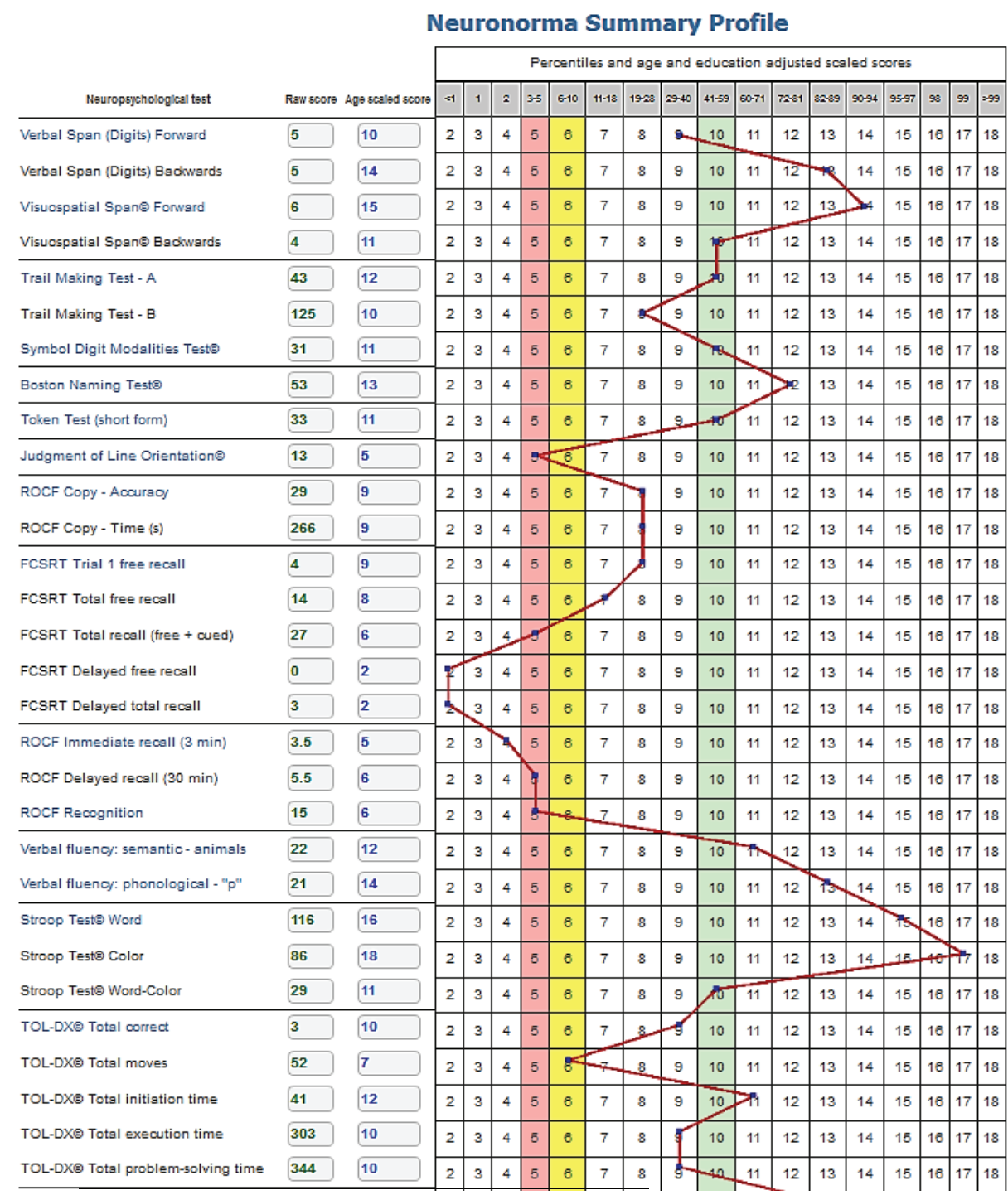

(B)

Artificial Neural Network: Probability of diagnosis of Alzheimer's disease

Click on the following button to transfer the test's values to the Neural Network
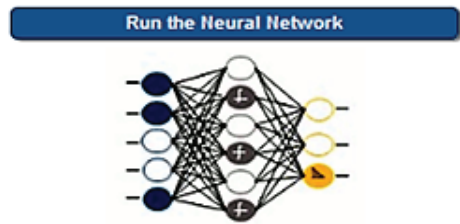

Normal Aging: $47.75 \%$

MCl: 49.69\%

Dementia: $02.55 \%$

Figure 1: (A) Screenshot of a sample neuropsychological profile, (B) Delivered probabilities by the implemented ANN. 


\section{Acknowledgements}

Members of the NEURONORMA.ES Study Team:

Principal Investigators: J.P.-C., Hospital de Mar, Barcelona, Spain; R Blesa, Hospital de la Santa Creu i Sant Pau, Barcelona, Spain; M. Aguilar., Hospital Mútua de Terrassa, Terrassa, Spain; J. L. Molinuevo., Hospital Clínic, Barcelona, Spain; A. Robles, Hospital Clínico Universitario, Santiago de Compostela, Spain; M. S. Barquero (deceased), Hospital Clínico San Carlos, Madrid, Spain; C. Antunez, Hospital Virgen Arrixaca, Murcia, Spain; C. Martinez-Parra., Hospital Virgen Macarena, Sevilla, Spain; A. Frank-García, Hospital Universitario La Paz, Madrid, Spain; M. Fernández, Hospital de Cruces, Bilbao, Spain.

Clinicians, Psychologists, and Neuropsychologists: Nina Gramunt (coordinator), Peter Böhm, Sonia González, Yolanda Buriel, María Quintana, Sonia Quiñones, Gonzalo Sánchez, Rosa M. Manero, Gracia Cucurella, Institut Municipal d'Investigació Mèdica. Barcelona, Spain; Eva Ruiz, Mónica Serradell, Laura Torner, Hospital Clínic. Barcelona, Spain; Dolors Badenes, Laura Casas, Noemí Cerulla, Silvia Ramos, Loli Cabello, Hospital Mútua de Terrassa, Terrassa, Spain; Dolores Rodríguez, Clinical Psychology and Psychobiology Department, University of Santiago de Compostela, Spain; María Payno, Clara Villanueva, Hospital Clínico San Carlos, Madrid, Spain; Rafael Carles, Judit Jiménez, Martirio Antequera, Hospital Virgen Arixaca, Murcia, Spain; Jose Manuel Gata, Pablo Duque, Laura Jiménez, Hospital Virgen Macarena, Sevilla, Spain; Azucena Sanz, María Dolores Aguilar, Hospital Universitario La Paz, Madrid, Spain; Ana Molano, Maitena Lasa, Hospital de Cruces, Bilbao, Spain.

\section{References}

[1] Amato, F., López, A., Peña-Méndez, E. M., Vaňhara, P., Hampl, A., \& Havel, J. (2013). Artificial neural networks in medical diagnosis. Journal of Applied Biomedicine, 11, 47-58.

[2] Benton, A. L., Sivan, A. B., Hamsher, K. S., Varney, N. R., \& Spreen, O. (1994). Contributions to neuropsychological assessment. New York, NY: Oxford University Press.

[3] Buschke, H. (1984). Cuedrecallin amnesia. Journal of Clinical Neuropsychology, 6, 433-440.

[4] Buscema, M. P., Grossi, E., Snowdon, D., Antuono, P., Intraligi, M., Maurelli, G., et al. (2004). Artificial Neural Networks and Artificial Organisms Can Predict Alzheimer Pathology in Individual Patients Only on The Basis of Cognitive and Functional 
Status. Neuroinformatics, 2, 399-416.

[5] Culbertson, W. C., \& Zillmer, E. A. (2001). Tower of London. Drexel University. TOLDX. North Tonawanda, NY: Multi-Health Systems.

[6] De Renzi, E., \& Faglioni, P. (1978). Development of a shortened version of the Token Test. Cortex, 14, 41-49.

[7] Di Luca, M., Grossi, E., Borroni, B., Zimmermann, M., Marcello, E., Colciaghi, F., et al. (2005). Artificial neural networks allow the use of simultaneous measurements of Alzheimer Disease markers for early detection of the disease. Journal of Translational Medicine, 3, 1-7.

[8] Golden, C. J. (1978). Stroop Color and Word Test. Chicago, IL: Stoeling.

[9] Gumbau, G. (2018). Programació del sistema de comunicacions entre dos servidors per aplicacions biomèdiques. Barcelona: Universitat Politèctica de Catalunya.

[10] Kaplan, E., Fein, D., Morris, R., \& Delis, D. (1991). WAIS-R as a neuropsychological instrument. San Antonio, TX: The Psychological Corporation.

[11] Kaplan, E., Goodglass, H., \& Weintraub, S. (2001). The Boston Naming Test (2nd ed.). Philadelphia, PA: Lippincott Williams \& Wilkins.

[12] Lezak, M. D., Howieson, D. B., Bigler, E. D., \& Tranel, D. (2012). Neuropsychological Assessment (Fifth ed.). New York: Oxford University Press, Inc.

[13] Osterrieth, P. A. (1944). Le test de copie d'une figure complexe: Contribution è l'étude de la perception et la mémoire. Archives de Psychologie, 30, 286-356.

[14] Partington, J., \& Leiter, R. (1949). Partington's pathways test. The Psychological Service Center Bulletin, 1, 9-20.

[15] Peña-Casanova, J. (2005). Programa Integrado de Exploración Neuropsicológica. Test Barcelona-Revisado. Barcelona: Masson. [Integrated program of neuropsychological assessment- Revised Barcelona Test].

[16] Peña-Casanova, J., Blesa, R., Aguilar, M., Gramunt-Fombuena, N., Gómez-Ansón, B., Oliva, R., et al. (2009). Spanish Multicenter Normative Studies (NEURONORMA Project): methods and sample characteristics. Archives of Clinical Neuropsychology, 24, 307-319.

[17] Peña-Casanova, J., Quiñones-Úbeda, S., Quintana-Aparicio, M., Aguilar, M., Badenes, D., Molinuevo, J.L., et al. (2009). Spanish Multicenter Normative Studies (NEURONORMA Project): norms for verbal span, visuospatial span, letter and number sequencing, trail making test, and symbol digit modalities test. Archives of Clinical Neuropsychology, 24, 321-341.

[18] Peña-Casanova, J., Quiñones-Úbeda, S., Gramunt-Fombuena, N., Aguilar, M., Casas, L., Molinuevo, J.L., et al. (2009). Spanish Multicenter Normative Studies 
(NEURONORMA Project): norms for Boston naming test and token test. Archives of Clinical Neuropsychology, 24, 343-354.

[19] Peña-Casanova, J., Quiñones-Úbeda, S., Gramunt-Fombuena, N., Quintana-Aparicio, M., Aguilar, M., Badenes, D., et al. (2009). Spanish Multicenter Normative Studies (NEURONORMA Project): norms for verbal fluency tests. Archives of Clinical Neuropsychology, 24, 395-411.

[20] Peña-Casanova, J., Gramunt-Fombuena, N., Quiñones-Úbeda, S., SánchezBenavides, G., Aguilar, M., Badenes, D., et al. (2009). Spanish Multicenter Normative Studies (NEURONORMA Project): norms for the Rey-Osterrieth complex figure (copy and memory), and free and cued selective reminding test. Archives of Clinical Neuropsychology, 24, 371-393.

[21] Peña-Casanova, J., Quiñones-Úbeda, S., Gramunt-Fombuena, N., Quintana-Aparicio, M., Aguilar, M., Molinuevo, J.L., et al. (2009). Spanish Multicenter Normative Studies (NEURONORMA Project): norms for the Stroop color-word interference test and the Tower of London-Drexel. Archives of Clinical Neuropsychology, 24, 413-429.

[22] Peña-Casanova, J., Quintana-Aparicio, M., Quiñones-Úbeda, S., Aguilar, M., Molinuevo, J.L., Serradell, M., et al. (2009). Spanish Multicenter Normative Studies (NEURONORMA Project): norms for the visual object and space perception batteryabbreviated, and judgment of line orientation. Archives of Clinical Neuropsychology, 24, 355-370.

[23] Peña-Casanova, J., Sánchez-Benavides, G., de Sola, S., Manero-Borrás, R. M., \& Casals-Coll, M. (2012). Neuropsychology of Alzheimer's Disease. Archives of Medical Research, 43, 686-693.

[24] Petersen, R. C., Smith, G. E., Waring, S. C., Invik, R. J., Tangalos, E. G., \& Kokmen, E. (1999). Mild cognitive impairment: Clinical characterization and outcome. Archives of Neurology, 56, 303-308.

[25] Quintana, M., Guàrdia, J., Sánchez-Benavides, G., Aguilar, M., Molinuevo, J. L., Robles, A., et al. (2012). Using artificial neural networks in clinical neuropsychology: High performance in mild cognitive impairment and Alzheimer's disease. Journal of Clinical and Experimental Neuropsychology, 34, 195-208.

[26] Reitan, R. M., \& Wolfson, D. (1993). The Halstead-Reitan neuropsychological test battery. Theory and clinical interpretation (2nd ed.). Tucson, AZ: Neuropsychology Press.

[27] Rey, A. (1941). L'examen psychologique dans les cas d'encéphalopathie traumatique. Archives de Psychologie, 28, 286-340. 
[28] Rivera, N. (2016). Predicció de la demència tipus Alzheimer mitjançant xarxes neuronals a partir de dades cognitives. Barcelona: Universitat Politèctica de Catalunya.

[29] Smith, A. (1973). Symbol Digit Modalities Test Manual. Los Angeles: Western Psychological Services.

[30] Warrington, E. K., \& James, M. (1991). Visual Object and Space Perception Battery. Suffolk: Thames Valley Test Co.

[31] Winblad, B., Palmer, K., Kivipelto, M., Jelic, V., Fratiglioni, L., Wahlund, L.O., et al. (2004). Mild cognitive impairment-beyond controversies, towards a consensus: report of the International Working Group on Mild Cognitive Impairment. Journal of Internal Medicine, 256, 240-246. 